\title{
Le théâtre de Rodrigo Garcia : un autoportrait à la dynamite
}

Bernadette Bost

\section{OpenEdition}

Journals

Édition électronique

URL : http://journals.openedition.org/recherchestravaux/381

DOI : 10.4000/recherchestravaux.381

ISSN : 1969-6434

Éditeur

UGA Éditions/Université Grenoble Alpes

\section{Édition imprimée}

Date de publication : 30 décembre 2009

Pagination : 103-110

ISBN : 978-2-84310-159-5

ISSN : 0151-1874

Référence électronique

Bernadette Bost, " Le théâtre de Rodrigo Garcia : un autoportrait à la dynamite », Recherches \& Travaux [En ligne], 75 | 2009, mis en ligne le 30 juin 2011, consulté le 08 septembre 2020. URL : http://

journals.openedition.org/recherchestravaux/381; DOI : https://doi.org/10.4000/recherchestravaux. 381

(c) Recherches \& Travaux 


\section{Le théâtre de Rodrigo Garcia : un autoportrait à la dynamite}

En s'annonçant constituée de "49 fragments, 3 listes, I8 dessins", Jardinage humain témoigne du goût de son auteur, Rodrigo Garcia, pour les formes hétérogènes et éclatées. Le fait est que toutes ses pièces ${ }^{\mathrm{I}}$, aussi bien que ses spectacles, sont marqués par la discontinuité de la forme, la juxtaposition de matériaux que rien ne lie : bribes de récits, réflexions morales entrecoupées d'envolées lyriques, listes de personnalités déclarées idéales ou exécrables, recettes de cuisine, éloges du football, anathèmes politiques et diatribes contre la société de consommation. Les mises en scène de ses pièces que signe le dramaturge - qui prend d'ailleurs les plus grandes libertés dans le traitement de ses textes, les coupant et les déstructurant - ne sont pas moins composites et génériquement impures, mêlant la danse et la performance, le sketch pornographique et la vidéo, l'enchaînement de monologues et le duo ou trio clownesque.

Leur rapport avec l'autobiographie est à peine moins ostensiblement affiché : les titres peuvent être écrits à la première personne (J'ai acheté une pelle chez Ikea pour creuser ma tombe), et le paratexte liminaire promet à l'occasion «un récit fragmenté d'événements personnels» comme dans Vous êtes tous des

I. Euvres de R. Garcia dont les traductions françaises sont publiées par les Solitaires intempestifs, à Besançon, dans la traduction de Chr. Vasserot : Vous êtes tous des fils de pute, 2001; Notes de cuisine, 2002; After sun, suivi de L'Avantage avec les animaux, c'est qu'ils t'aiment sans te poser de questions, 2002; Fallait rester chez vous, têtes de noud, 2002; Borges, 2002; L'Histoire de Ronald, le clown de McDonald's, suivi de J'ai acheté une pelle chez Ikea pour creuser ma tombe, 2003; Jardinage humain, 2003; Agamemnon, 2004; Goya, 2006.

Chez un autre éditeur : Notes de cuisine, avec une préface de M. Martinez Thomas, Presses universitaires du Mirail, 1998. 
fils de pute, pièce dont une des parties se veut en effet «biographique, comme les pages libres d'un journal intime». Promesse fiable, affiche crédible, "je» non fictif, volonté attestée de faire entendre la voix de l'auteur et d'utiliser sa vie même comme matière à fiction? Il n'est pas inutile de tester la part de l'«écriture de soi» dans les œuvres de Rodrigo Garcia, avant même d'y chercher un véritable autoportrait.

Dans un entretien avec une journaliste de L'Humanité ${ }^{2}$, le dramaturge et metteur en scène livre quelques clés quand il raconte :

J'ai grandi dans la banlieue de Buenos Aires et c'est un apprentissage de l'humilité. Les garçons, à I4 ans, savent qu'ils seront ouvriers à l'usine ou dans le bâtiment. Leur avenir est parfaitement tracé. Moi-même, j'étais destiné à prendre la succession de mon père et hériter de sa boucherie. J'ai grandi dans la rue mais j'ai pu poursuivre des études, fréquenter l'université et j'ai commencé à lire, à lire énormément.

\section{Famille, amis, victimes}

Ce n'est pas un hasard si la compagnie de Rodrigo Garcia s'appelle Carniceria Teatro (le Théâtre boucherie). Garcia fait référence dans Borges (2002) au commerce familial quand il évoque ses parents, "mon père boucher et ma mère marchande des quatre-saisons» (p. I9). Même si Monique Martinez Thomas, dans une préface à Notes de cuisine, présente plutôt ses parents comme des "émigrés asturiens passionnés d'art dramatique et propriétaires, à Grand Bourg, d'une charcuterie», les références au métier de boucher sont multiples dans ces textes, et les «je» se montrent très au fait du travail de la viande, avec autant de technicité que de brutalité.

Au-delà de ce cercle familial, Garcia fait surgir à tout propos, sous leur vrai nom, des personnes réelles qui comptent pour lui. C'est le cas par exemple, dans Goya, du philosophe allemand Peter Sloterdijk, dont il a déclaré dans une interview qu'il aimait lire les œuvres. Il n'a pu qu'apprécier le Traité de la raison cynique publié par ce penseur en 1987 : Garcia se réclame volontiers - ou du moins ses locuteurs, ses «je» le font-ils - de Diogène et du cynisme philosophique. Ce qui ne l'empêche pas de présenter l'homme Sloterdijk sous un jour pas particulièrement flatteur dans Goya: il en fait une star qui ne consent à se déplacer que si on la paie grassement ("la secrétaire de cet enfoiré nous dit que Sloterdijk veut huit briques pour venir en Espagne»), mais qui se laisse finalement tenter par l'appât de "croquettes à se taper le cul par terre, chez Casa Manolo» (p. 33).

2. «Je fais un théâtre sale, laid», propos recueillis par Zoé Lin, L’Humanité, I8 juillet 2002. 
C'est surtout dans Borges, en fait, sa pièce certainement la plus autobiographique en dépit de quelques dérapages délirants dans l'imaginaire, qu'abondent les noms de personnes de son entourage au moment où le locuteur est supposé aller dynamiter la tombe genevoise de l'écrivain argentin :

Je vais aller saccager la tombe du vieux Borges à Genève.

Oui $[\ldots]$ : je me mets en route dans ma Fiat Punto grise qui ne m'a jaaaaamais lâché, et je vais dire à Oscar et à Delphine et à Macasdar de m’aider, de me laisser dormir chez eux et de me trouver de la dynamite, des pelles et de quoi MANGER.

Et je vais appeler mon éditeur, mon ami François qui est de Besançon, c'est juste à côté, pour qu'il publie tout ça.

Je vais y aller avec Patricia, avec Miguelito et avec Chete, pour qu' ils gueulent des tas d'insultes pendant que j'explose la tombe. (p. 35)

On a là une rafale de références à la réalité. La tombe de Borges se trouve bien à Genève, au cimetière de Plainpalais - tombe $n^{\circ} 735$, rue des Rois - et le prénom Oscar désigne Oscar Gomez Mata, metteur en scène d'origine argentine installé dans la cité helvétique où il a monté la pièce de Garcia Boucher espagnol en 1997, au théâtre Saint-Gervais. L'une de ses actrices s'appelle Delphine. Macasdar (Philippe) dirige le théâtre Saint-Gervais, et François n'est autre que François Berreur, directeur à Besançon des Solitaires intempestifs et donc éditeur des traductions françaises des œuvres du dramaturge. Sans oublier la présence d'une Patricia, d'un Miguel et d'une Chete dans la troupe du Carniceria Teatro.

Certes, de telles références ne garantissent pas l'authenticité des faits relatés dans une fiction, et tout "je» ne renvoie évidemment pas à l'auteur. Il faut prendre avec circonspection ce que le locuteur dit de ses parents, par exemple, y compris dans les textes à caution autobiographique comme Borges, quand il prodigue des anecdotes données comme des souvenirs de sa vie adolescente, notamment la relation de son retour à la maison après la rencontre d'Octavio Paz et Jorge Luis Borges dans un café de Buenos Aires, l'année de ses 17 ans :

J'arrive à la maison - chez mon père et ma mère, mon père boucher et ma mère marchande des quatre-saisons - et je leur dis, tout ému : je les ai vus, Borges et Octavio Paz. Ma mère me pose des questions. Elle me demande si je leur ai parlé. Elle sait que je les admire. Mon père me flanque un coup de ceinture, il m’engueule en me traitant de pédé [...] et cinq minutes plus tard je me retrouve avec un tablier blanc plein de sang en train de découper du bøuf à la boucherie. (p. 19)

Ce qui n'empêche pas le même «je» d'affirmer, dans un autre texte, que ses parents ne l'ont jamais touché... Les «raclées» paternelles (subies ici par Garcia fils ou prétendument infligées par Garcia papa dans Agamemnon) sont 
des excroissances artistiques chez un auteur qui travaille sur l'excès comme d'autres sur la litote : on peut les comparer avec les pseudo-confidences sur ses méthodes d'éducation de père qui juge plus formateur de faire boire de l'anisette à son fils de bon matin (dans Notes de cuisine) que de l'envoyer à l'école. Il y a d'ailleurs une jubilation à évoquer la "raclée» chez Rodrigo Garcia : il consacre à ce thème tout un monologue de Fallait rester chez vous, têtes de noud:

Filer des raclées.

Oui : filer des raclées. Des raclées. Des coups de batte.

Ça oui, c'est de la raclée. De la bonne. [...]

Une bonne raclée, bien comme il faut. Une raclée. Une tournée. J'arrive et je colle une raclée et avant de partir, je dis au-revoir à coups de raclées. Je pars d'un endroit en collant une raclée et j'arrive autre part en collant une autre raclée. [etc.] (p. 7)

\section{Souvenirs et fantasmes}

En fait, tout l'art de Rodrigo Garcia consiste à se déplacer sans cesse entre vérité et fiction, livrant un instant son vrai visage pour mieux jouer l'instant d'après avec les masques. Dans la recherche des indices de vérité, le lecteur est contraint d'utiliser sa propre intuition, ce qui n'est peut-être pas si éloigné qu'on pourrait le croire d'une démarche scientifique, car l'intuition procède d'une synthèse d'un ensemble de connaissances sur une ouvre et son auteur. On peut aussi se référer (moins scientifiquement?) à la vraisemblance d'un certain nombre de "confidences", comme celles qui concernent sa mère et leur commune position sociale :

Ma mère [...] rit parce que j'intitule une pièce de théâtre CONNAÎTRE DES GENS, BOUFFER DE LA MERDE 3 . Elle rit - ET MOI IL ME REND TRÈS HEUREUX LE RIRE DE MA MÈRE - et elle lâche : «Avec tout ce qui t'est arrivé de bien dans la vie, comment tu peux choisir un titre pareil.» Mais elle rit, et à son rire je comprends toute sa frustration, je sais que dans le fond elle est d'accord avec moi, elle m'autorise à être le porte-parole d'une génération de perdants qui se sont fait enculer jusqu'à l'os. (Borges, p. 19-2I)

Il est également fort tentant, à la lecture de Borges, de croire à l'émoi de ce jeune homme qui a rencontré Borges, son idole. Rappelons-nous sa confidence à Zoé Lin dans l'interview citée plus haut : «j'ai commencé à lire, énormément». Ce qui incite à croire qu'il a bien entrepris, adolescent, de lire tout Schopenhauer parce que Borges aimait Schopenhauer, et que la

3. Notons au passage que Connaître des gens, bouffer de la merde est effectivement le titre d'une pièce de Rodrigo Garcia, de 1999. 
rencontre du maître l'a mis dans tous ses états - même si le dramaturge exagère probablement en déclarant qu'il s'est "pissé dessus». Est vraisemblable encore le récit de son désarroi quand, lors d'une conférence, il a osé interroger Borges sur Schopenhauer et que le maître a répondu en utilisant un mot inconnu de lui : «Schopenhauer, c'est le pinacle»... D'autant que le héros ( $s$ 'il est permis de l'appeler ainsi), de déconvenue en déconvenue, a fini par prendre la décision d'émigrer sous d'autres cieux, ce que Rodrigo Garcia a fait effectivement en 1986 :

Je vais me casser.

Je vais me casser.

Je vais me casser en Espagne ou à Madagascar.

Et finalement je me casse. (p. 39-4I)

Reste que ce même héros a exprimé de façon un peu excessive, auparavant, sa frustration de ne pas comprendre le mot "pinacle» :

[...] ça fait quatre ans que j'étudie Schopenhauer, je veux être brillant, je veux parler avec Borges devant trois millions de personnes et il me répond avec un mot que je ne comprends pas.

Je vais l'attendre à la sortie pour lui flanquer une bonne raclée.

Je vais lui mettre un coup de bifteck.

Un coup de gigot.

Des gens prennent sa défense, je leur flanque des coups de gigot.

Le chien d'aveugle qu'il a toujours après lui essaie de me mordre, je tue le chien à coups de poing... qu'est-ce que je dis : Borges ne s'est jamais baladé avec un chien, peu importe...

Je lui donne un coup sur le front avec l'os de mon gigot, il y a du sang partout. (p. 29)

Le fantasme contamine encore allègrement la réalité quand le locuteur déclare qu'il fait semblant d'être aveugle comme Borges pour séduire les filles, qu'il s'achète même un chien d'aveugle, ou quand il affirme avoir tiré un coup de feu sur sa mère, quitte à corriger aussitôt :

J'ai jamais tiré sur personne, je me suis pas non plus acheté le chien, ni la canne, j'ai pas joué les aveugles pour baiser, j'ai pas eu les couilles, parce que, si ça avait été le cas, je ne dirais pas ce que je suis en train de dire, je tiendrais ma langue. (p. 2I-23)

Ce qui ne l'empêche pas de se représenter plus loin en train de dynamiter la tombe de Borges avec une telle violence que les restes du "Vieux» (ainsi l'appelle-t-il) volent "jusqu'à l'obélisque» qui est "dans le centre de Buenos Aires»! Lui-même n'en revient pas : "Jusqu'à l'obélisque, impossible : c'est pas la porte à côté, c'est [...] sur un autre contineeeeeennnnt!» Et non seulement les pauvres restes atteignent-ils l'obélisque, mais on les retrouve même "devant la porte $7 \mathrm{du}$ stade de Boca», là où l'«on vend les sandwiches de 
chorizo, les choripains!». Et voilà les «cendres du vieux Borges» sur les braises où grille le chorizo. Et le vieux «se fait bouffer en plein match» de foot alors que c'est «ce qu'il détestait le plus, bordel, le foot!»

\section{Stratégie de la confusion}

S'agit-il là d'un simple dynamitage rêvé des restes de Borges - et à travers lui d'un establishment littéraire argentin - ou d'un dynamitage de soi dans un autoportrait qui se plaît à lancer dans toutes directions les fragments contradictoires, impossibles à rassembler, d'une identité éclatée? Fragments, d'abord, du Rodrigo Garcia de 17 ans, éperdu d'admiration pour la grande littérature, convaincu de la valeur supérieure des grands écrivains; fragments, ensuite, du Rodrigo Garcia de 35 ans qui porte un tout autre regard sur ses anciennes idoles :

Je l'ai vu au café Tortoni, Borges, avec sa secrétaire et son secrétaire et avec Octavio Paz, le poète qui ne s'est jamais mouillé pour rien ni personne, le poète décoré, le poète médaille. Ils étaient assis là, les deux poètes médaille, ceux qui ne se sont jamais mouillés pour personne, et tout au fond des inconnus jouaient au billard.

Mais moi, je ne les voyais pas de cet œeil. Avec mes dix-sept ans et une vocation littéraire, je les voyais telles deux apparitions. [...] Dix-sept ans! À cet âge-là, on ne sait pas ce qu'on admire. (p. 15)

Et ce sont aussi les morceaux disjoints et opposés d'un «moi» qui, de pièce en pièce, se montre non seulement adolescent et adulte, fils et père, mais aussi homme et femme, sincère et menteur, ami des chiens et torturant des animaux, un cynique (au sens non philosophique, cette fois) capable de désespoir et de fureur devant les malheurs des hommes. Dans Jardinage humain apparaissent nettement ces différents éclats d'identité comme autant de masques. La tentation cynique, par exemple, associée aux hésitations permanentes du velléitaire, s'expose dans le dernier des monologues, consacré à la recherche de la bonne formule de réussite artistique, et débité par un jeune acteur nu comme la Vérité même :

Je veux être artiste, alors je dois choisir. Parce qu'un artiste, soit il est au fond du trou, soit il avance à fond la caisse.

L'un ou l'autre, mais à fond.

Être à fond : à fond la caisse ou au fond du trou.

Tu vas bien : d'accord, mais tu y vas à fond.

Tu vas mal: d'accord, mais tu y vas à fond.

Ce qu'on ne peut pas faire, si on veut être artiste, c'est passer inaperçu. Vivre en demi-teinte, ne jamais y aller à fond.

$\mathrm{Si}$ tu vis comme tout le monde, tu ne seras jamais reconnu comme artiste. (p. 29) 
Le tout est de se garder de toute mesure, de "mettre le paquet", ce qui est la condition sine qua non, affirme le locuteur, pour «se faire remarquer au théâtre». Excès de débauche ou de déprime, peu importe du moment que l'excès est la règle, et «Pas besoin d'avoir quelque chose à dire pour être artiste, ce qu'il faut c'est une vie publique qui dépasse les bornes.» Autrement dit, «si je fais dans l'excès, j'aurai du succès" (p. 30). Déclaration troublante pour le spectateur qui ne peut que constater la mise en œuvre dans tous les travaux de Garcia, en effet, d'une esthétique de l'excès. En dépit de ce cynisme, cet homme de théâtre ne se prive pas de dénoncer les responsables du malheur social, au point qu'on est tenté de lui prêter par moments une infinie compassion pour l'humanité souffrante. Ses manifestations de compassion elles-mêmes, toutefois, ne sont pas dénuées d'ambiguïté comme on peut le constater dans le film vidéo sur le thème des pietà diffusé dans sa mise en scène de Jardinage humain : une même figure assise, les cheveux longs recouverts d'un capuchon qui cache les traits du visage - une femme, mais pourquoi ne pas y reconnaître un double féminin de Garcia - tient dans ses bras une succession de corps inertes tels des christs morts, des gens de tous âges et de tous milieux, du cadre supérieur au camionneur, de la mère de famille au père Noël de supermarché, dans toute sorte d'environnements contemporains. Où se manifeste la plus décapante ironie, cette figure de style consistant à dire le contraire de ce qu'on veut faire entendre : dans ces pietà qui font rire le public parce qu'elles sont déplacées dans des cadres insolites, ou dans la déclaration provocatrice de l'artiste-écrivain déclarant que son "excès» n'est rien qu'un choix de marketing?

À défaut de répondre à cette question, nous ne pouvons que prendre acte de la somme des passions divergentes proclamées d'une pièce à l'autre : pour Quevedo et le trash, la boxe et l'opéra, la cuisine et les Saint-Sébastien de la peinture ancienne... Et constater qu'avec le pouvoir médiumnique des artistes, Rodrigo Garcia se trouve en empathie avec les aspirations multiples et contradictoires de ses contemporains, d'où la litanie (scandaleuse?) des "Je veux être" dans After sun (p. 19-20), suite d'identités idéales d'acteurs, chanteurs, animaux de dessins animés, footballeurs, monstres du cinéma, figures de saints (plus Jésus et Judas), boxeurs, compositeurs, cinéastes, génies et célébrités en tout genre, grands écrivains, héros bibliques, dieux ou déesse (Aphrodite), et aussi dictateurs (Staline, Franco, Pinochet), sans oublier Karol Wojtyla, le maire de Paris, etc., jusqu'à l'idole des idoles Diego Maradona qu’il imagine même peint par Vélasquez ou Goya jusque dans «la plus noire des peintures noires".

Â ce point, l'autoportrait éclaté de Rodrigo Garcia semble trouver son apothéose, dans After sun, en une sorte de "portrait de l'artiste en Maradona", 
somme des aptitudes à toutes les jouissances, tous les abus, tous les conformismes et toutes les trahisons assumées, dans ce "désordre d'extrême limite» qui lui permettrait d'atteindre un comble d'humanité :

Je veux être comme Diego Maradona, vivre une vie de débauche, défendre la tradition et en même temps l'assaisonner d'huile et de sel et dévorer le tout, démolir la Terre entière rien que pour moi, cracher sur tout ce qui m'entoure, me vidanger la vessie n'importe où, et être le plus humain de tous, de tous, de tous. (p. 2I)

Comble d'humanité ou comble de cynisme, encore une fois? À moins qu'il n'y ait une intention politique dans l'étalage des aspirations contraires d'un «je» saisi du vertige de la confusion généralisée? Lors du colloque de Rennes sur les «mises en scène du monde», en 2005, Rodrigo Garcia a évoqué tout ce qui l'irrite dans le comportement de ses contemporains et l'incurie des politiques, pour développer en conclusion une stratégie personnelle d'engagement négatif :

Lorsque je dis qu'en tant qu'artiste je suis conscient de cette décevante réalité, je ne prétends pas être plus sensible ou perspicace qu'un autre. Je me vois plutôt comme un Candide qui doit savoir porter un tel fardeau, qui est le noyau de sa passion artisanale : je suis un artisan de la contestation, je m'évertue à engendrer du malaise et, en même temps, des étincelles de beauté. Et je me sens dans l'obligation de semer la confusion ${ }^{4}$.

Au risque de brouiller l'autoportrait et d'interdire toute reconstitution d'une image présentable.

4. Mises en scène du monde, actes du colloque international de Rennes, Besançon, Les Solitaires intempestifs, 2005, p. 384. 